\title{
Cat-Inspired Deep Convolutional Neural Network for Bone Marrow Cancer Cells Detection
}

\author{
R. Kavitha ${ }^{1, *}$ and N. Viswanathan ${ }^{2}$ \\ ${ }^{1}$ Department of ECE, Bharathiyar Institute of Engineering for Women, Deviyakurichi, 636112, India \\ ${ }^{2}$ Department of ECE, Mahendra Engineering College, Namakkal, 637503, India \\ *Corresponding Author: R. Kavitha. Email: kavitharbe@gmail.com \\ Received: 19 August 2021; Accepted: 06 December 2021
}

\begin{abstract}
Bone marrow cancer is considered to be the most complex and dangerous disease which results due to an uncontrolled growth of white blood cells called leukocytes. Acute Lymphoblastic Leukemia (ALL) and Multiple Myeloma (MM) are considered to be important categories of bone cancers, which induces a larger number of cancer cells in the bone marrow, results in preventing the production of healthy blood cells. The advent of Artificial Intelligence, especially machine and deep learning, has expanded humanity's capacity to analyze and detect these increasingly complex diseases. But, accurate detection of cancer cells and reducing the probability of false alarm rates remain on the darker side of the research. This paper proposes novel deep convolutional neural networks in which the hyper parameters are optimized by adaptive Multi-objective CAT algorithms. The proposed model is trained on the preprocessed cell images followed by training with Optimized Convolutional Neural Network (OCNN) and finally detecting the category of cancer cells present in the bone marrow. The extensive experimentations have been carried out using SN-AM datasets and various performance metrics such as accuracy, precision, recall, specificity, and F1-score are calculated and analyzed. The overall accuracy was found to be $99.45 \%$ in predicting different categories of cancer and it outperforms the pre-trained deep learning models such as Alexnets, VGG-16 nets, and U-nets. To establish the superiority of the proposed model, we have compared the performance of the proposed model with other machine learning models such as Support Vector Machines, Random Forest, Naïve Bayes, and Artificial Neural Networks. From the above intensive studies, it is clear that the proposed model was able to produce brighter performances than the other learning models in detecting the category of cancer cells.
\end{abstract}

Keywords: Bone marrow cancer; leukocytes; multiple myeloma; acute lymphoblastic leukemia; multi-objective cat; optimized convolutional neural networks

This work is licensed under a Creative Commons Attribution 4.0 International License, which permits unrestricted use, distribution, and reproduction in any medium, provided the original work is properly cited. 


\section{Introduction}

The unerring blood cell discerning and enactment plays an essential role in the screening and prognosis of several chronic disease like sepsis [1], immune system disorders [2], blood cancer [3]. The blood cells are of three different types made incessant in the bone marrow and emancipates at the correct time in the blood stream. Sometimes, the natural growth of the blood cell is impeded by the augmented growth of anomalous blood cells is the foremost cause of blood cancer. There are different types of blood cancers, among that leukemia, myeloma and lymphoma are deadly chronic cancer. The clinicians, doctors have disparate phases of cognition for each kind of leukemia and judgement of all white blood cells is not similar may produce grave errors [4]. The white blood cancer epitome leads to blistering in the bone marrow is called Acute Lymphocytic Leukemia (ALL).

The patient blood is prognoses with deem to the immunophenotypic, morphological, cytogenetic facets for diagnosing the chronic diseases [5], the elementary inspection in the premature stage is by diagnosing through microscopic investigation of stained blood smears procured from peripheral blood/bone marrow. In the conventional diagnosis, the clinicians develop a slide of blood-smeared, fix and spattered the slide with chemical foreign agents like Hematoxylin-Eosin and Wright-Giemsa stains, heeded by the sedulous observation of any changes in the blood cell and count of the cell. The count of the blood cell is regular in the normal healthy condition, indeed if leukemia, it is observed to be lower blood count due to anomalous unhealthy cell inhabited in the space of RBCs. Roughly, 1500 patients died of bone erosion which contributes $0.2 \%$ to chronic deadly cancer types in 2019 [6].

It is surveyed that 20,000 people prognosis in US for blood cancer every year. The treatment for this chronic disease relayed on the age, epitome of the cancer and its progressing speed and cancer blistering areas [7]. Thereby, the blood count plays the primary factor in distinction of cancer epitome. The blood cell counting can be done either manually or automatically. If it is done manually by skilled physician, it leads in $100 \%$ appreciation rate but it takes long time-consuming process [8]. This method needs not only time, labor and its associated cost. It is liable to the mutability of staining quality that relay on the spattering of chemical agents of the skilled physician [9].

The autonomous method of counting the blood cells is the rapid process with greater risk of the wrong counts. This method is cost effective and garrison it on urban, rural areas simply. The issues on manual count of blood cells are invaded through the propounded method may cause inconsistencies due to classification of the labor manually, need of skilled physician, the errors on observing under microscope. At present, there are several methods overcomes these issues. The epitome of leukemia depends on the proportion of the white blood cells under visual image analysis and processing [10].

The several label free procedures are diagnosis the blood cells have been reconnoitered to do perform the process on biological samples. The machine learning schemes can maneuver sustained uniform standards on very high-dimensional datasets to enact label-free image classification [11,12]. Singh et al. [13] necessitate manual extraction of features by label free image classification using machine learning do not fully utilize the high-complexity data of three-dimensional data which gives more accuracy. Deep Learning scheme comes to the role to manifest preferable functioning than typical Machine Learning conventional method for processing a large number of images [14]. Deep learning schemes can succor all the inducted challenges because they inherit desirable attributes from the raw data themselves [15]. Convolutional Neural network $(\mathrm{CNN})$ is category of deep learning algorithm which finds its best place in detection of bone marrow cancer cells. Even though several CNN algorithms [16] are used, still high false alarm rate and handling the larger data remains to be a critical issues. To overcome this issue, this paper proposes a robust and intelligent detection framework based on optimized convolutional neural network for better classification of bone marrow cancer cells. 


\section{Related Works}

Uma Rani et al. proposed the machine learning based artificial neural network algorithm for predicting the cancer at bone marrow in childhood and teens. ANN is used efficiently for data regression, prediction. Visual Gene Developer's NeuralNet class is inherited for "Projected Principal-Edge Distribution" (PPED) feature recognition algorithm for extracting the features and then its edges of white blood cells are classified using classification algorithm. The experimentation on the blood test from whole blood exemplars from six outpatients is compared with the existing some methods. It performs better feature extraction and classification than the compared method [16].

Rodellar et al. focuses on image processing and machine learning for the morphological characterization and autonomous identification of cell images obtained from peripheral blood smears. The practical experimentation was addressed the segmentation of the whole 12 cell groups Propounded with the number of 16,408 images from 374 patients were obtained with the CellaVision DM96 in the Core Laboratory of the Spain Hospital Clinic of Barcelona inherited with the resolution of $360 \times 363$ pixels. The accuracy obtained was high on soft clustering using Gaussian mixture models. It helps in analyzing the better interpret on several morphological features and serve as better learning on survey tools [17].

Salah et al. surveyed on the ML trending techniques for its diagnosis of all types of leukaemia. The 58 articles of full text were reviewed with the inclusive of 22 studies discussing ALL, AML, CLL, and CML was $12,8,3$, and 1 accordingly. Most of the $70 \%$ studies had small and homogenous samples are diagnosed using supervised learning for classification tasks. Thus, this review demonstrated widely the need to evolve further the field of ML research, inclusive of the transformation from significant designing algorithms to practically applying them clinically [18].

Chandradevan et al. describes the start-up steps concerning the evolution of a machine learning digital pathology mechanism. It performs differential cell count (DCC) and classifying all non-neoplastic bone marrow cellular constituents of the DCC and performs many test cases on multiple myeloma (MM). The propounded software inherited by the system will attain good accuracy in detecting the cell and classifying phases by two stage system relayed on the convolution neural network. It attains applauding performance in classification and detection tasks [19].

Brück et al. assessed the myelodysplastic syndrome (MDS) and myeloproliferative neoplasm (MPN), bone marrow (BM) histopathology using machine learning based CNN. The CNN inherits the morphologic attributes from "236_MDS, 87_MDS/MPN, and 11_control BM biopsies". These attributes identified the genetic-cytogenetic aberrations, prognosis, age, and gender in multivariate regression models. Then mutation prediction probability is identified to correlate with variant allele frequency. Thus the results demonstrating that the propounded algorithms had the potential to recognize the various morphologic patterns [20].

Jiang et al. constructs the WBCNet model that extract the features of the microscopic white blood cell image fully by amalgamates with the batch normalization algorithm, residual convolution architecture along with the improved activation function. This propounded model has 33 layers, whereas speed was high compared to the traditional methods, thereby identifies the epitome of white blood cell. In this work, "92800_white blood cell images and 9200_images" are used for data augmentation. The count of "81600 images \& 20400 images comprise of training set and the test set. WBCNet reached the highest accuracy rate when training to 110-130 times epoch. Thus, the work assists the doctors to prognosis on leukaemia, and decreases the misdiagnosis rate [21].

Tabe et al. thrives on an automated diagnostic support mechanism using MDS and autonomous blood cell image-recognition system using a deep learning system (DLS) empowered by convolutional neural networks (CNNs) with a decision-making system relays on extreme gradient boosting (XGBoost). The experimentation deals with datasets consisting of 695,030 blood cell images for training set. It is obtained 
from 3,261 PB smears with inclusive of hematopoietic malignancies. The DLS parallelly classified 17 blood cell types and 97 morphological features. Thus, the results revealed that the autonomous MDS diagnostic system significantly discriminates the MDS from aplastic anemia (AA) with considerable better accuracy [22].

Ryu et al. develops a white blood cell classification framework that captures the three dimensional (3D) refractive index using deep learning. With 3D RI distributions is utilized for every white blood cell for exploitation in accurate profiling of their subtypes. The deep learning empowered in our propounded method inherits the high-dimensional information of the WBC RI tomogram voxels and attained the high accuracy. Therefore, the results outstand on cost effective prognosis of hematologic malignancy [23].

$\mathrm{Wu}$ et al. propounds the BMSNet based on deep learning algorithm for evaluating the single nuclear balls morphologies in bone marrow smears with rapid process and accurate monitoring. The deep learning method uses cell relayed performance was correlated with the hematologist performance. The results obtained with intra variant among the specialist participated, since it is operator dependent [24].

Kumar et al. employs the deep learning technique for omitting the probability of errors causes in the manual workflow by employing convolutional neural networks. It utilizes the optimized Dense Convolutional neural network framework DCNN for detecting the cancer epitome. The accuracy obtained was higher than the conventional machine learning method produces the accurate results for 94 times on $100[25]$.

\section{Proposed Framework}

Fig. 1 shows complete architecture for the proposed framework. The working mechanism of the proposed deep learning based classification system is sub-divided into three important phases. Data preparation, Data augmentation process, segmentation and feature extraction using deep convolutional layers and finally trained by the novel cat optimized fully connected trained networks.

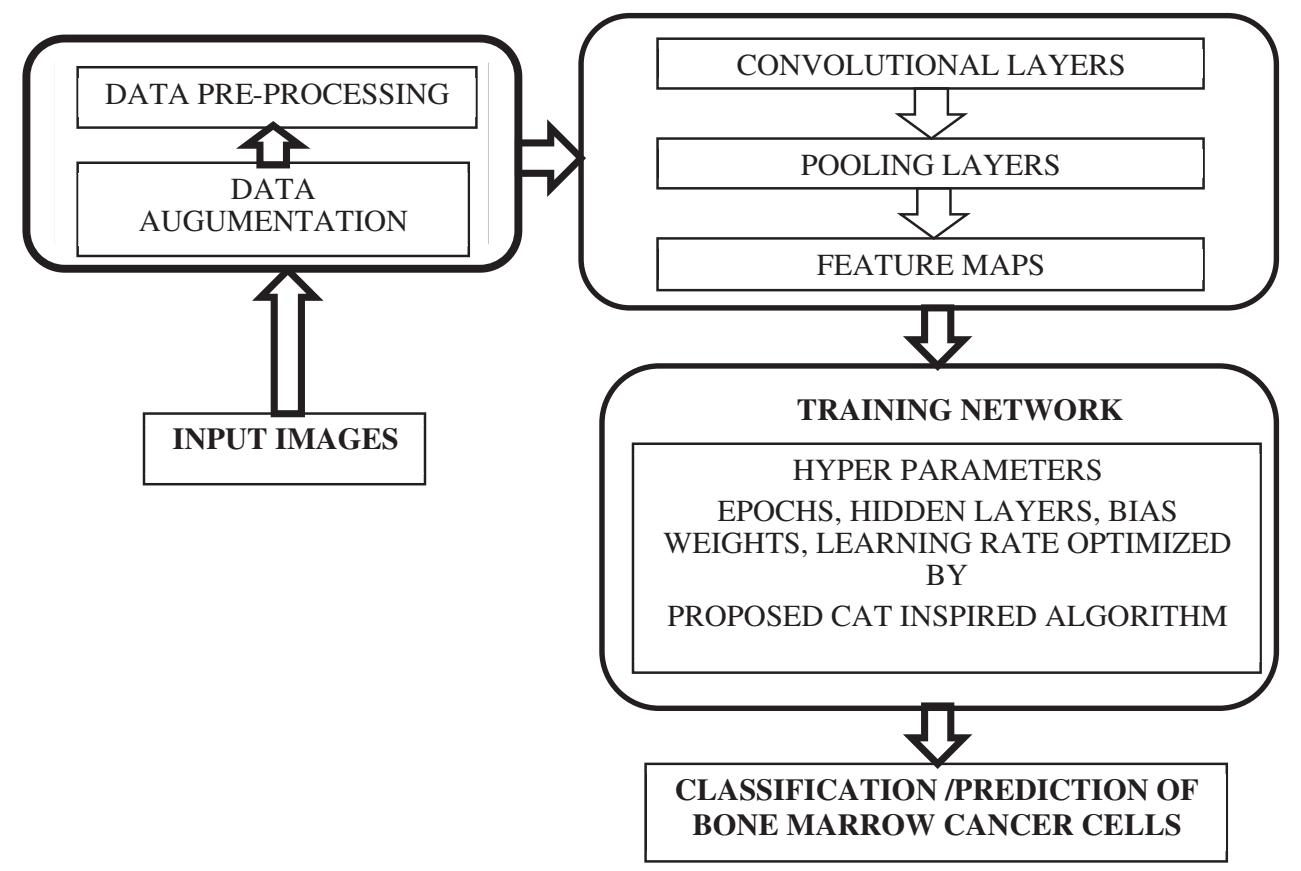

Figure 1: Complete architecture for the proposed framework 


\subsection{Data Preparation Process}

Microscopic images were captured from bone marrow aspirate slides of patients diagnosed with Blineage Acute Lymphoid Leukemia (B-ALL) and Multiple Myeloma (MM) as per the standard guidelines. Slides were stained using Jenner-Giemsa stain. Images were captured at $1000 \times$ magnification using Nikon Eclipse-200 microscope equipped with a digital camera. Images were captured in raw BMP format with a size of $2560 \times 1920$ pixels. In all, this dataset consists of 90 images of B-ALL and 100 images of MM. Both MM and B-ALL images have sufficient variability from one image to another image to rigorously test any stain normalization methodology developed. The detailed descriptions are given as [13].

\subsection{Data Augmentation Process}

After collection of the input images, image augmentation process is used in the proposed architecture. The training models based on neural networks leads to the overfitting problems where a limited quantity of labelled data is available. The most proficient and efficient method to tackle this problem is data augmentation. During the data augmentation phase, each image undergoes a series of transformation, producing the huge amount of newly correlated training image samples. Affine transformation is employed for an efficient data augmentation. The affine transformation techniques such as translation, scaling and rotations are used. Mostly the training image samples obtained from the augmentation process have a correlation, this step is recommended to overcome the overfitting problems. Fig. 2 shows the different cancer cell images after the data augmentation process.

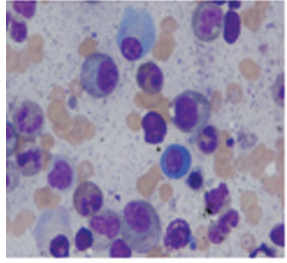

(a)

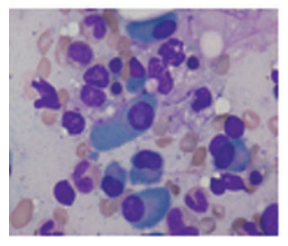

(d)

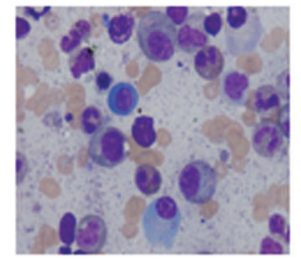

(b)

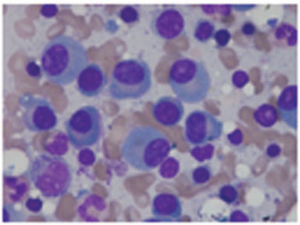

(e)

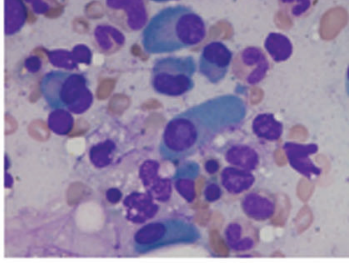

(c)

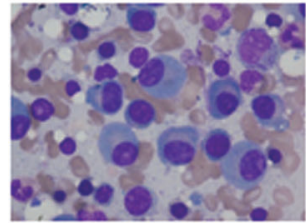

(f)

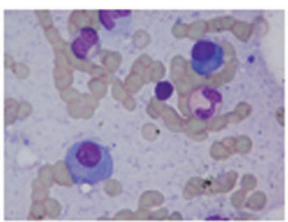

(g)

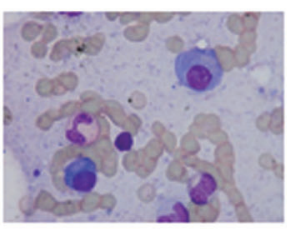

(h)

Figure 2: Augmented cancer cell images (a-d) MM cancer cell images, (e-h) ALL cancer cell images

\subsection{Proposed Training Methodology}

This section discusses about the convolutional neural network and cat optimization algorithm used for tuning the hyper parameters. 


\subsubsection{Convolutional Neural Networks}

The CNN architecture is composed of a different layers such as Convolutional layers (CL), Pooling Layers (PL), Fully Connected layers (FCL) and Output layers (OL). The CL layers are responsible for extracting the different features from the input cancer cell images using various convolutional filters. These convolutional operation is performed by these filters at every offset of the input images. The features extracted from the $\mathrm{CL}$ are concatenated and mapped into feature space using non-linear activation unit (reLu). The PL is used to reduce the dimensions of the feature maps obtained from the CL and retain the most addressable information of the image. In the proposed architecture, Max-Pooling Layers are used for retaining the information of the image. The last layer of $\mathrm{CNN}$ is fully connected layer that classifies the extracted feature maps into a particular class. To reduce the misclassification rate, CNN training involves the adjustments of the parameters of convolutional kernels and hidden neurons in the fully connected layers. To achieve this, stochastic gradient descent (SGD) training method is used to tune the CL and fully connected layer parameters. The major drawback of implementing SGD is that it contains many hyper parameters that may impact on network's performance. Fig. 3 shows the overview architecture of convolutional neural network.

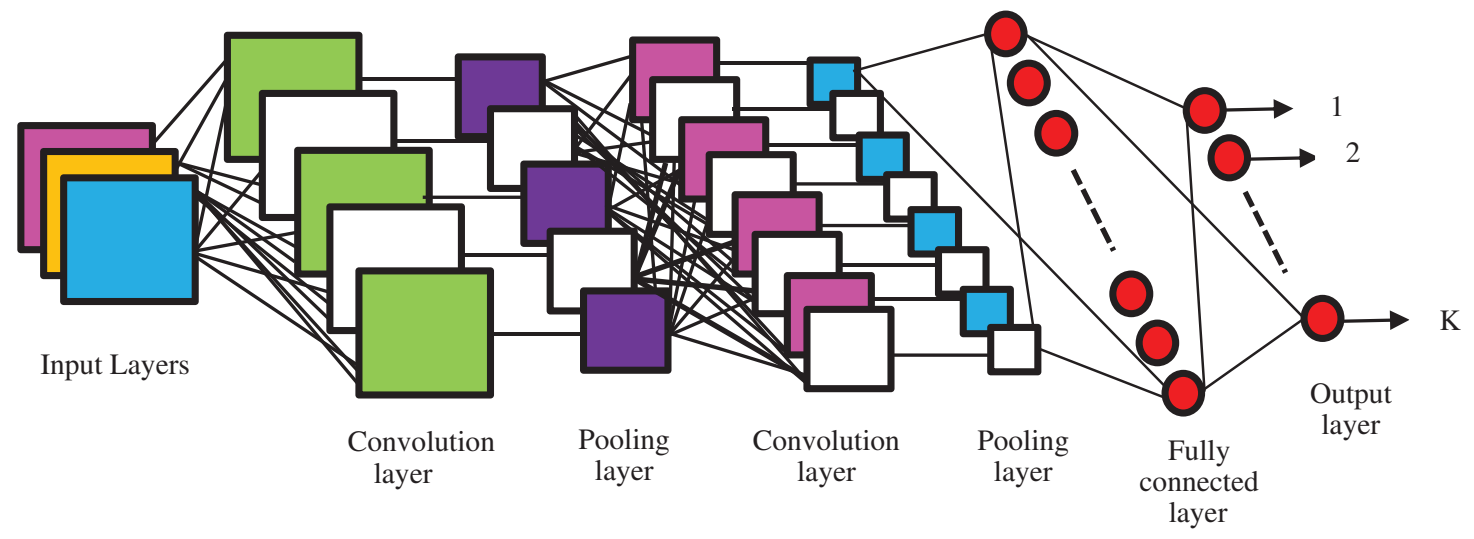

Figure 3: Convolutional neural network and its functioning layers

Motivated by the drawbacks of SGD and its impact on network performance, the proposed architecture uses the CAT-Inspired algorithms used for the optimization mechanism. Moreover, the details of the CNN layers used in the proposed architecture is depicted in Tab. 1. The working mechanism of the CAT optimized on training network is discussed in preceding section.

Table 1: Parameters for $\mathrm{CNN}$ used in the proposed architecture

\begin{tabular}{llll}
\hline Sl. No. & No of convolutional layers & Output size & Stride length \\
\hline 1 & Conv(2d)_Layer-1 & 64 & $1 \times 1$ \\
2 & Max-Pooling layers-1 & 0 & $2 \times 2$ \\
3 & Conv(2d)_Layer-2 & 64 & $1 \times 1$ \\
4 & Max-Pooling layers-2 & 0 & $2 \times 2$ \\
5 & Conv(2d)-Layer-3 & 32 & $1 \times 1$ \\
6 & Max-Pooling layers-3 & 0 & $2 \times 2$ \\
\hline & & & (Continued)
\end{tabular}


IASC, 2022, vol.33, no.2

\begin{tabular}{llll}
\hline \multicolumn{2}{l}{ Table 1 (continued) } \\
\hline S1. No. & No of convolutional layers & Output size & Stride length \\
\hline 7 & Conv(2d)_Layer-4 & 32 & $1 \times 1$ \\
8 & Max-Pooling layers-4 & 0 & $2 \times 2$ \\
9 & Conv(2d)-Layer-5 & 16 & $1 \times 1$ \\
10 & Max-Pooling layers-5 & 0 & $2 \times 2$ \\
11 & Fully connected layers & 02 & $1 \times 1$ \\
\hline
\end{tabular}

\subsubsection{Cat Optimization Algorithm}

The original cat swarm optimization is a continuous and single-objective algorithm which is inspired by resting and tracing behaviors of cats. Cats seem to be lazy and spend most of their time resting. However, during their rests, their consciousness is very high and they are very aware of what is happening around them. So, they are constantly observing the surroundings intelligently and deliberately and when they see a target, they start moving towards it quickly. Therefore, CSO algorithm is modeled based on combining these two main deportments of cats. The CSO algorithm is composed of two modes, namely, tracing and seeking modes. Each cat represents a solution set, which has its own position, a fitness value, and a flag. The position is made up of $\mathrm{M}$ dimensions in the search space, and each dimension has its own velocity; the fitness value depicts how well the solution set (cat) is; finally, the flag is to classify the cats into either seeking or tracing mode. Thus, we should first specify how many cats should be engaged in the iteration and run them through the algorithm. The best cat in each iteration is saved into memory, and the one at the final iteration will represent the final solution. Fig. 4 illustrates the working flow of CAT swarm optimization algorithm. The working mechanism of seeking and tracing modes are discussed in following section.

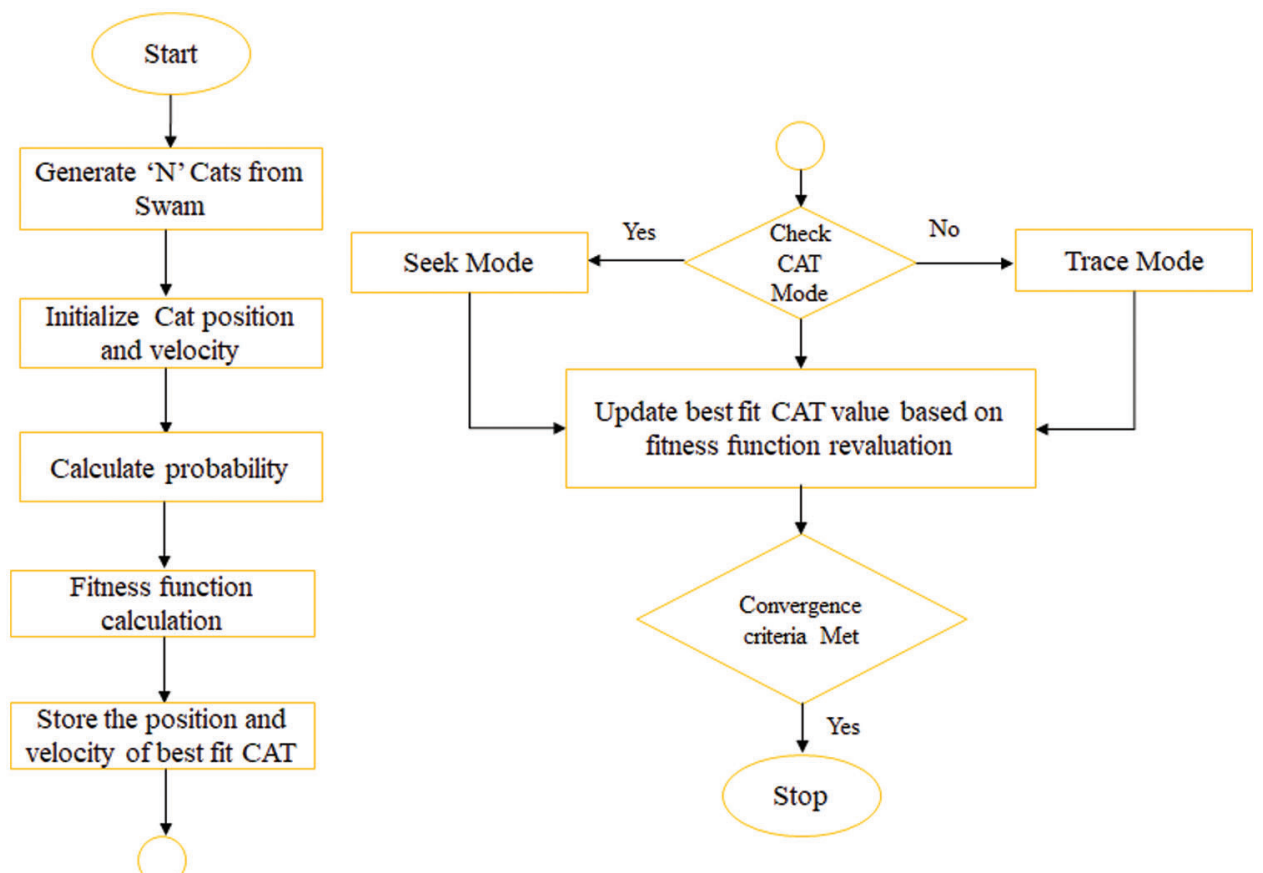

Figure 4: Complete working diagram for the cat swarm optimization algorithm 


\section{Seeking Modes}

This mode imitates the resting behavior of cats, where four fundamental parameters play important roles: seeking memory pool (SMP), seeking range of the selected dimension (SRD), counts of dimension to change (CDC), and self-position considering (SPC). These values are all tuned and defined by the user through a trial-and-error method.

SMP specifies the size of seeking memory for cats, i.e., it defines number of candidate positions in which one of them is going to be chosen by the cat to go to, for example, if SMP was set to 5, then for each and every cat, 5 new random positions will be generated and one of them will be selected to be the next position of the cat. How to randomize the new positions will depend on the other two parameters that are CDC and SRD. CDC defines how many dimensions to be modified which is in the interval of $[0,1]$. For example, if the search space has 5 dimensions and CDC is set to 0.2 , then for each cat, four random dimensions out of the five need to be modified and the other one stays the same. SRD is the mutative ratio for the selected dimensions, i.e., it defines the amount of mutation and modifications for those dimensions that were selected by CDC. Finally, SPC is a Boolean value, which specifies whether the current position of a cat will be selected as a candidate position for the next iteration or not. So, for example, if the SPC flag is set to true, then for each cat, we need to generate (SMP-1) number of candidates instead of SMP number as the current position is considered as one of them. Seeking mode steps are as follows

(1) Make as many as SMP copies of the current position of Catk.

(2) For each copy, randomly select as many as CDC dimensions to be mutated. Moreover, randomly add or subtract SRD values from the current values, which replace the old positions as shown in the following Eq. (1).

$x($ new_cat $)=(1+$ rand $+S R D) * x($ old_cat $)$

where $\mathrm{x}$ (new_cat) is new cat's new position, $\mathrm{x}$ (old_cat) is cat's initial position and rand is random interval of time between $[0,1]$.

(3) Calculate the Fitness function (FF) and based on probability, candidate position is selected based on the highest fitness function as shown in following expression (2)

$P(i)=\mid\left(F F(i)-F F(b) \mid /\left(F F_{\max }-F F_{\text {min }}\right)\right.$

where $\mathrm{FF}(\mathrm{i})$ is fitness of current cat $\mathrm{FF}(\mathrm{b})$ is no of cat population, FFmax is Maximum Fitness Function, FFmin is the Minimum Value of Fitness function.

\section{Tracing Modes}

This mode copies the tracing behavior of cats. For the first iteration, random velocity values are given to all dimensions of a cat's position. However, for later steps, velocity values need to be updated. Moving cats in this mode are as follows:

(i) Update velocities (V (CAT)) for all dimensions according to below Eq. (3)

$V(C A T)=V(C A T)+r * c(x($ new_cat $)-x($ old_cat $))$

where a and c are constants.

The complete working mechanism of the proposed CAT-Inspired algorithm is depicted in Fig. 4.

\subsubsection{Hyper Parameter Optimized Using Cat Algorithm}

In this proposed model, the hyper parameters in fully connected layers are optimized using CSO. Since these hyper parameters plays a crucial role in determining network's performance, selecting hyper parameter is essential and depends on the application for which CNN is implemented. The hidden layers, input weights, 
number of epochs, learning rate are considered to be the most common hyper parameter setting in the CNN. The significance of these hyper parameters are depicted in Tab. 2.

Table 2: Hyper parameter and its functionalities

\begin{tabular}{|c|c|c|}
\hline Sl. No. & Hyper parameters & Functionalities \\
\hline 01 & Learning Rate & Parameter used to control the speed of training network \\
\hline 02 & Epochs & $\begin{array}{l}\text { Represents the number of times the learning model updates the } \\
\text { network according to the datasets }\end{array}$ \\
\hline 03 & $\begin{array}{l}\text { Hidden Layers and } \\
\text { Input weights }\end{array}$ & Decides the working flow of the model \\
\hline
\end{tabular}

To yield more accurate results, these hyper parameters are need to be optimized. The proposed CATinspired optimization of hyper parameters in CNN's SGD is given in Algorithm 1. The cat populations are selected randomly based on the number of input weights, hidden layers, learning rate and number of epochs. The new fitness function is formulated by modifying the equation

Fitness Function $A=\{1-A(i)\}$

where A is Accuracy, A(i) is the accuracy at $i^{\text {th }}$ new iteration

The details of the optimized hyper parameters obtained after the implementation of proposed optimization algorithm is presented in Tab. 3 .

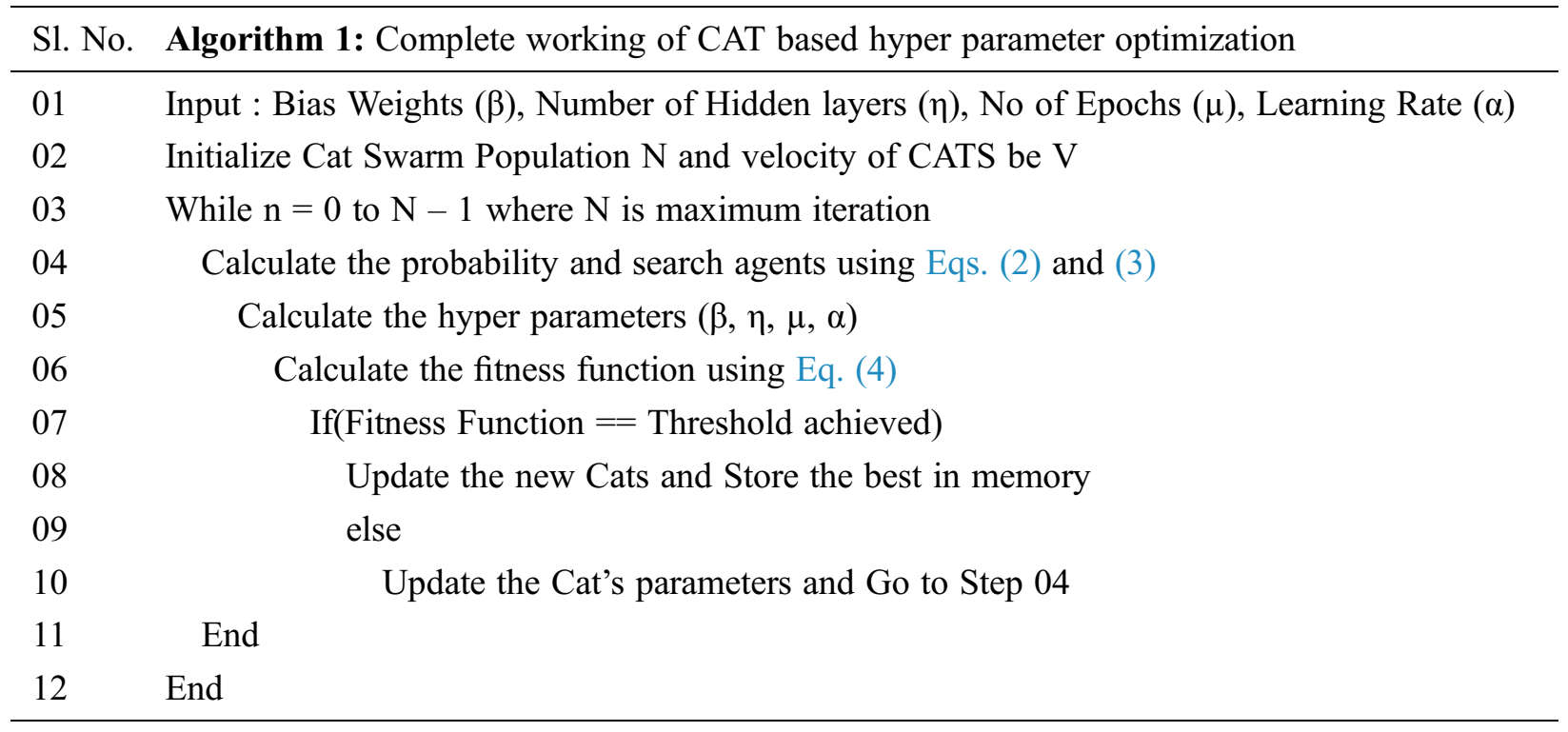


Table 3: Optimized hyper parameters used for training the network in CNN

\begin{tabular}{lll}
\hline S1. No. & Hyper parameters & Optimized hyper parameters \\
\hline 01 & Batch size & 20 \\
02 & No of epochs & 20 \\
03 & Learning rate & 0.015 \\
04 & Number of optimized iterations & 45 \\
05 & No of search agents & 30 \\
\hline
\end{tabular}

\section{Results and Discussion}

\subsection{Implementation Details}

The proposed model is implemented using Tensorflow v.18, an end to end python open source platform. The multi-classification model was trained on 1024 images in 1000 iterations. The CAT optimizer was used to optimize the loss functions in the networks which yields minimum loss during the iteration. The trained model was then used for identifying the bone marrow cancer in the images. The model was trained on i7 CPU, 16 GB RAM, NVIDIA K80 GPU with 2.5 GHz Operating Frequency.

The subsequent section describes the results of the proposed model. The comparative analysis of the proposed model with state-of-art learning models are also discussed.

\subsection{Performance Metrics}

In this section, we have shown the superiority of the proposed model over the other deep learning models. The total number of datasets used for training and testing the proposed model is depicted in Tab. 4.

Table 4: Total number of datasets (after augmentation) used for training and testing the proposed network

\begin{tabular}{llll}
\hline Sl. No. & Total number of images & Training data (\%) & Testing data (\%) \\
\hline 01 & 1024 & 70 & 30 \\
\hline
\end{tabular}

Four Convolutional Layers are used in the proposed and hyper parameters of the proposed model are optimized by Cat-Swarm Optimization. To evaluate the performance of proposed architecture, metrics such as accuracy, sensitivity, specificity, recall and fl-score are calculated. Tab. 5 shows the mathematical expressions for calculating the metrics used for evaluating the proposed architecture.

Table 5: Mathematical expressions for the performance metrics' calculation

\begin{tabular}{lll}
\hline S1. No. & Performance metrics & Mathematical expression \\
\hline 01 & Accuracy & $\frac{T P+T N}{T P+T N+F P+F N}$ \\
02 & Sensitivity or recall & $\frac{\mathrm{TP}}{\mathrm{TP}+\mathrm{FN}} \times 100$ \\
\hline
\end{tabular}


IASC, 2022, vol.33, no.2

\begin{tabular}{lll}
\hline \multicolumn{1}{c}{ Table 5 (continued) } & \\
\hline S1. No. & Performance metrics & Mathematical expression \\
\hline 03 & Specificity & $\frac{T N}{T N+F P}$ \\
04 & Precision & $\frac{T N}{T P+F P}$ \\
05 & F1-Score & $2 \cdot \frac{\text { Precision } * \text { Recall }}{\text { Precision }+ \text { Recall }}$ \\
\hline
\end{tabular}

Figs. 5 and 6 shows the validation curve of the proposed architecture in detecting the cancer categories. From the Fig. 7, it is found that the proposed architecture reaches its maximum accuracy after 400 iteration and remains constant till 1000 iteration. The similar fashion of performance is found from the Figure also. The ROC curves (Fig. 7) and confusion matrix (Fig. 8) of the proposed framework in detecting the categories of bone marrow cancers cells to test the classification effectiveness. Following the application of proposed methodology, 99.6\% is achieved in detecting the ALL type of cancers, $99.57 \%$ is achieved in detecting the MM type of cancers. In the non-optimized CNN layers, $92.0 \%$ and $92.1 \%$ accuracy is achieved in detecting the both types of cancer respectively. Further it is observed nearly $5 \%$ increase in performance of classification is achieved.

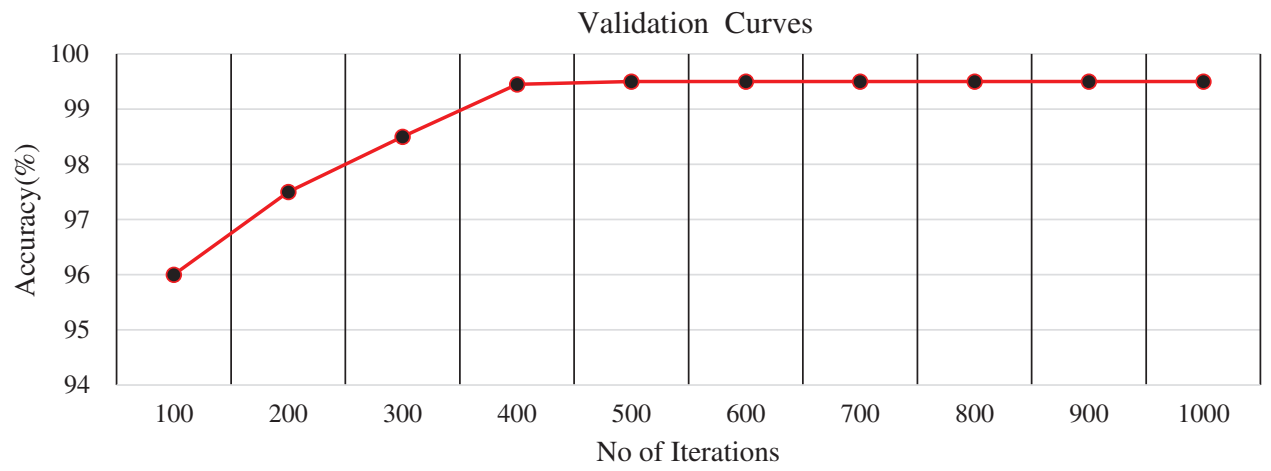

Figure 5: Validation curve for the proposed architecture in detecting the ALL type of bone cancers

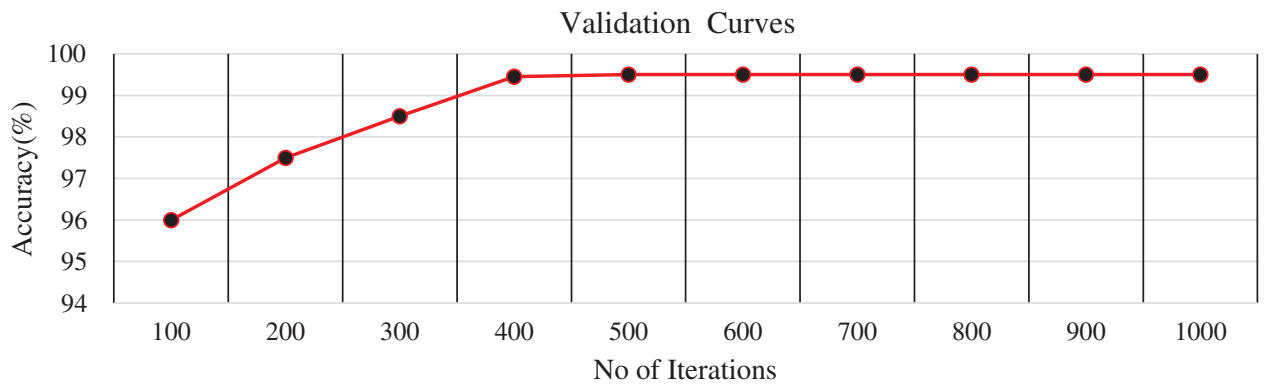

Figure 6: Validation curve for the proposed architecture in detecting the MM type of bone cancers 


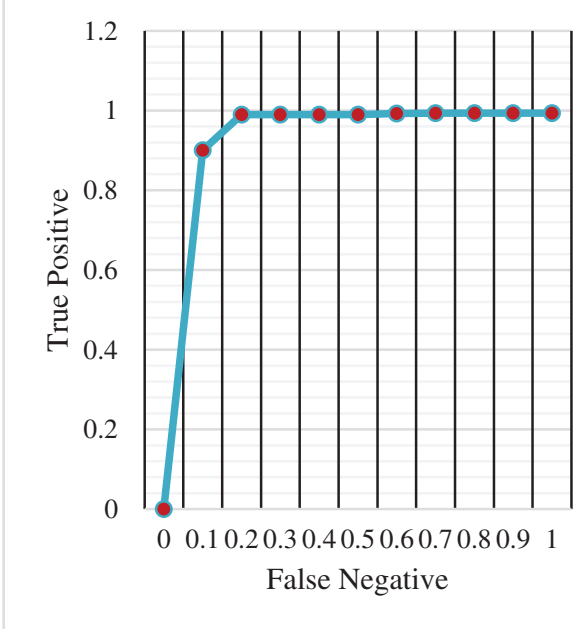

(a)

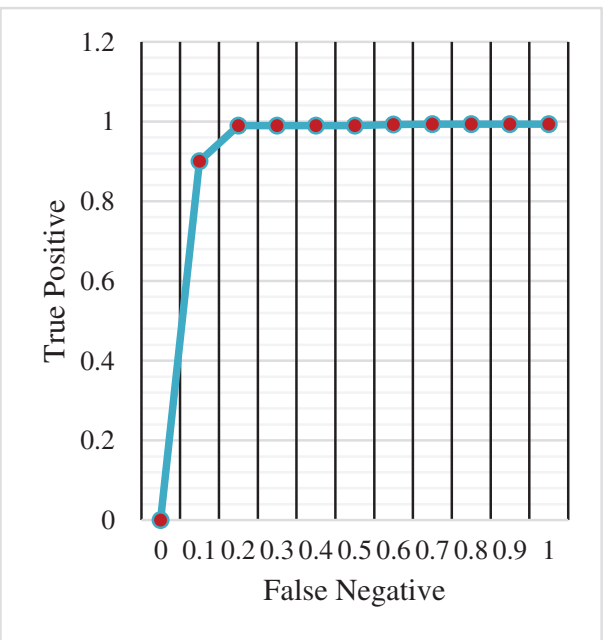

(b)

Figure 7: ROC curves for the proposed algorithm. (a) In detecting ALL type of cancer, (b) In detecting MM type of cancers

\begin{tabular}{|l|l|l|l|}
\hline CLASSES & ALL & MM & NORMAL \\
\hline ALL & $\mathbf{3 4 1}$ & $\mathbf{0}$ & $\mathbf{1}$ \\
\hline MM & $\mathbf{1}$ & $\mathbf{3 4 0}$ & $\mathbf{1}$ \\
\hline NORMAL & $\mathbf{1}$ & $\mathbf{2}$ & $\mathbf{3 3 9}$ \\
\hline
\end{tabular}

(a)

\begin{tabular}{|l|l|l|l|}
\hline CLASSES & ALL & MM & NORMAL \\
\hline ALL & $\mathbf{2 8 1}$ & $\mathbf{3 0}$ & $\mathbf{2 9}$ \\
\hline MM & $\mathbf{2 9}$ & $\mathbf{2 8 0}$ & $\mathbf{3 1}$ \\
\hline NORMAL & $\mathbf{3 1}$ & $\mathbf{2 9 3}$ & $\mathbf{2 8 1}$ \\
\hline
\end{tabular}

(b)

Figure 8: Confusion matrixes (a) Proposed architectures, (b) Non-optimized CNN architecture

Tabs. 6 and 7 presents the comparative analysis between the performances of proposed and CNN algorithms (without optimization). From the Tab. 6, it is found that proposed algorithm has shown the accuracy of $99.6 \%, 99.2 \%$ precision, $99.5 \%$ recall and high $\mathrm{fl}$ score of $99.89 \%$ in detecting the ALL type of cancer cells. Also it is observed the proposed algorithm has exhibited the similar performance of detecting the MM type of cancers. From the above Tabs. 6 and 7, it is clear that the proposed optimized algorithm has exhibited the better performance in detecting the cancer cells and also reduces computational complexity. 
IASC, 2022, vol.33, no.2

Table 6: Performance metrics for the CNN (without optimization) for the prediction of bone marrow cancers

\begin{tabular}{lllllll}
\hline \multirow{2}{*}{ S1. No. } & Type of cancer & \multicolumn{5}{c}{ Performance metrics (\%) } \\
\cline { 3 - 6 } & & Accuracy & Precision & Recall & Specificity & F1-Score \\
\hline 01 & ALL & $92.0 \%$ & $92.4 \%$ & $91.0 \%$ & $91.4 \%$ & $92.7 \%$ \\
02 & MM & $92.1 \%$ & $91.25 \%$ & $90.5 \%$ & $90.3 \%$ & $90.2 \%$ \\
\hline
\end{tabular}

Table 7: Performance metrics for the proposed algorithm for the prediction of bone marrow cancers

\begin{tabular}{|c|c|c|c|c|c|c|}
\hline \multirow[t]{2}{*}{ S1. No. } & \multirow[t]{2}{*}{ Type of cancer } & \multicolumn{5}{|c|}{ Performance metrics $(\%)$} \\
\hline & & Accuracy & Precision & Recall & Specificity & F1-Score \\
\hline 01 & ALL & $99.6 \%$ & $99.2 \%$ & $99.5 \%$ & $99.3 \%$ & $99.89 \%$ \\
\hline 02 & MM & $99.57 \%$ & $99.25 \%$ & $99.44 \%$ & $99.32 \%$ & $99.90 \%$ \\
\hline
\end{tabular}

\subsection{Comparative Analysis}

To establish the superiority of the proposed algorithm, we have compared the other existing models such Alexnets, U-nets, Resnets, VGGNET-16, Support vector machines (SVM), Naïve Bayes (NB), Random Forest (RF) and Artificial Neural Network (ANN).

Tabs. 8 and 9 presents the comparative analysis between the performances of proposed and existing algorithms. From the Tabs. 8 and 9, it is clear that optimization technique adopted in the proposed architecture has shown the superior performance than other existing algorithms in such a way $10 \%$ greater than Alexnets, $15 \%-16 \%$ greater than U-Nets, Resnets, $9 \%-10 \%$ greater than traditional $\mathrm{CNN}$, VGG-16 nets and even 15\% greater than other state-of-art learning models. Hence the proposed algorithm can find the better place than other the existing learning models in detection of cancer cells.

Table 8: Performance analysis of different algorithms for detection of ALL type of cancer cells

\begin{tabular}{lllllll}
\hline \multirow{2}{*}{ S1. No. } & \multirow{2}{*}{ Algorithm } & \multicolumn{4}{c}{ Performance metrics (\%) } \\
\cline { 3 - 6 } & & Accuracy & Precision & Recall & Specificity & F1-Score \\
\hline 01 & Alexnets & $90 \%$ & $89.5 \%$ & $88.5 \%$ & $88.45 \%$ & $89.7 \%$ \\
02 & U-Nets & $84.5 \%$ & $83.5 \%$ & $84.5 \%$ & $82.5 \%$ & $80.4 \%$ \\
03 & RESNETS & $85 \%$ & $84.3 \%$ & $83.5 \%$ & $84 \%$ & $83.2 \%$ \\
04 & VGG-16 & $91 \%$ & $91.5 \%$ & $92 \%$ & $91.45 \%$ & $92.4 \%$ \\
05 & CNN & $92.0 \%$ & $92.4 \%$ & $91.0 \%$ & $91.4 \%$ & $92.7 \%$ \\
06 & SVM & $78 \%$ & $78.45 \%$ & $77 \%$ & $76.5 \%$ & $75 \%$ \\
07 & NB & $70 \%$ & $68.5 \%$ & $75 \%$ & $74.5 \%$ & $74 \%$ \\
08 & RF & $68 \%$ & $67.5 \%$ & $67 \%$ & $68 \%$ & $67.5 \%$ \\
09 & ANN & $68.5 \%$ & $68.4 \%$ & $68.34 \%$ & $68.25 \%$ & $68 \%$ \\
10 & Proposed architecture & $99.6 \%$ & $99.2 \%$ & $99.5 \%$ & $99.3 \%$ & $99.89 \%$ \\
\hline
\end{tabular}


Table 9: Performance analysis of different algorithms for detection of MM type of cancer cells

\begin{tabular}{lllllll}
\hline \multirow{2}{*}{ S1. No. } & Algorithm & \multicolumn{4}{c}{ Performance metrics (\%) } \\
\cline { 3 - 6 } & & Accuracy & Precision & Recall & Specificity & F1-Score \\
\hline 01 & Alexnets & $90 \%$ & $89.5 \%$ & $88.5 \%$ & $88.45 \%$ & $89.7 \%$ \\
02 & U-Nets & $84.5 \%$ & $83.5 \%$ & $84.5 \%$ & $82.5 \%$ & $80.4 \%$ \\
03 & RESNETS & $85 \%$ & $84.3 \%$ & $83.5 \%$ & $84 \%$ & $83.2 \%$ \\
04 & VGG-16 & $91 \%$ & $91.5 \%$ & $92 \%$ & $91.45 \%$ & $92.4 \%$ \\
05 & CNN & $92.0 \%$ & $92.4 \%$ & $91.0 \%$ & $91.4 \%$ & $92.7 \%$ \\
06 & SVM & $78 \%$ & $78.45 \%$ & $77 \%$ & $76.5 \%$ & $75 \%$ \\
07 & NB & $70 \%$ & $68.5 \%$ & $75 \%$ & $74.5 \%$ & $74 \%$ \\
08 & RF & $68 \%$ & $67.5 \%$ & $67 \%$ & $68 \%$ & $67.5 \%$ \\
09 & ANN & $68.5 \%$ & $68.4 \%$ & $68.34 \%$ & $68.25 \%$ & $68 \%$ \\
10 & Proposed architecture & $99.57 \%$ & $99.25 \%$ & $99.44 \%$ & $99.32 \%$ & $99.90 \%$ \\
\hline
\end{tabular}

\section{Conclusion}

The proposed research is to detect and classify the types of bone marrow cancer cells using microscopic cancer images. To detect the type of cancer cells, this work uses the optimized hyper parameters to reduce the computational complexity which affects the performance of the training network, further the proposed architecture integrates the CAT-Optimized Hyper parameters in CNN training network for classification of images with the high accuracy and less computational overhead. The proposed algorithm was developed using Tensorflow 1.8 with Keras API and compared with other existing state-of-art deep and machine learning architectures. The clear comparisons has shown that the proposed architecture has outperformed the other state-of art architectures in detecting the different categories of bone marrow cancer cells such as ALL and MM. However, we have to admit the algorithm needs improvisation in terms of handling the different clinical real time data sets.

Funding Statement: The authors received no specific funding for this study.

Conflicts of Interest: The authors declare that they have no conflicts of interest to report regarding the present study.

\section{References}

[1] R. M. Bateman, M. D. Sharpe, M. Singer and C. G. Ellis, "The effect of sepsis on the erythrocyte," International Journal of Molecular Sciences, vol. 18, no. 9, pp. 1-23, 2017.

[2] H. von Boehmer and F. Melchers, "Checkpoints in lymphocyte development and autoimmune disease," Nature Immunology, vol. 11, no. 11, pp. 14-20, 2010.

[3] M. Sant, C. Allemani, C. Tereanu, R. De Angelis, R. Capocaccia et al., "Incidence of hematologic malignancies in Europe by morphologic subtype: Results of the HAEMACARE project," Blood, vol. 116, no. 19, pp. 3724-3734, 2010.

[4] S. H. Rezatofighi and H. Soltanianzadeh, "Automatic recognition of five types of white blood cells in peripheral blood," Computerized Medical Imaging Graphics, vol. 35, no. 4, pp. 333-343, 2011.

[5] D. Norris and J. Stone, "WHO classification of tumors of hematopoietic and lymphoid tissues," WHO Classification of Tumors, vol. 5, pp. 20-35, 2021. 
[6] R. L. Siegel, K. D. Miller, H. E. Fuchs and A. Jemal, "Cancer statistics, 2021," CA: A Cancer Journal for Clinicians, vol. 71, no. 1, pp. 7-33, 2021.

[7] Y. Liu and F. Long, “Acute lymphoblastic leukemia cells image analysis with deep bagging ensemble learning," in ISBI, 2019 C-NMC Challenge: Classification in Cancer Cell Imaging, Lecture Notes in Bioengineering. Singapore: Springer, pp. 113-121, 2019.

[8] A. B. Kul'chyns'kyi, V. M. Kyjenko, W. Zukow and I. L. Popovych, "Causal neuro-immune relationships at patients with chronic pyelonephritis and cholecystitis. Correlations between parameters EEG, HRV and white blood cell count," Open Medicine, vol. 12, no. 1, pp. 201-213, 2017.

[9] S. Kumar, S. H. Ong, S. Ranganath and F. Chew, "Invariant texture classification for biomedical cell specimens via non-linear polar map filtering," Computer Vision \& Image Understanding, vol. 114, no. 1, pp. 44-53, 2010.

[10] J. Jung, S. J. Hong, H. B. Kim, G. Kim, M. Lee et al., "Labelfree non-invasive quantitative measurement of lipid contents in individual micro algal cells using refractive index tomography," Scientific Reports, vol. 8, no. 1, pp. 10-17, 2018.

[11] Y. Ozaki, H. Yamada, H. Kikuchi, A. Hirotsu, T. Murakami et al., "Label-free classification of cells based on supervised machine learning of subcellular structures," PLoS One, vol. 14, no. 1, pp. e0211347, 2019.

[12] N. Nissim, M. Dudaie, I. Barnea and N. T. Shaked, "Real-time stain-free classification of cancer cells and blood cells using interferometric phase microscopy and machine learning," Journal of Quantitative Cell Science, vol. 99, no. 5, pp. 511-523, 2021.

[13] V. Singh, V. Srivastava and D. S. Mehta, "Machine learning-based screening of red blood cells using quantitative phase imaging with micro-Spectro calorimetry," Optics and Laser Technology, vol. 124, pp. 24-29, 2020.

[14] S. Mourya, S. Kant, P. Kumar, A. Gupta and R. Gupta, "Leukonet: DCT-based CNN architecture for the classification of normal versus leukemic blasts in b-all cancer," Computer Vision and Pattern Recognition, arXiv preprint arXiv: 1810.07961, 2018.

[15] I. Arel, D. C. Rose and T. P. Karnowski, "Deep machine learning-A new frontier in artificial intelligence research [Research Frontier]," IEEE Computational Intelligence Magazine, vol. 5, no. 4, pp. 13-18, 2010.

[16] V. Uma Rani and B. Ramya, "Childhood bone marrow and artificial neural network," Journal of Computer Engineering, vol. 5, pp. 58-60, 2020.

[17] J. Rodellar, S. Alférez, A. Acevedo, A. Molina and A. Merino, "Image processing and machine learning in the morphological analysis of blood cells," International Journal of Laboratory Hematology, vol. 40, pp. 4653, 2018.

[18] H. T. Salah, I. N. Muhsen, M. E. Salama, T. Owaidah and S. K. Hashmi, "Machine learning applications in the diagnosis of leukemia: Current trends and future directions," International Journal of Laboratory Hematology, vol. 41, no. 6, pp. 717-725, 2019.

[19] R. Chandradevan, A. A. Aljudi, B. R. Drumheller, N. Kunananthaseelan, M. Amgad et al., "Machine-based detection and classification for bone marrow aspirate differential counts: Initial development focusing on nonneoplastic cells," Laboratory Investigation, vol. 100, no. 1, pp. 98-109, 2020.

[20] O. E. Brück, S. E. Lallukka-Brück, H. R. Hohtari, A. Ianevski, F. T. Ebeling et al., "Machine learning of bone marrow histopathology identifies genetic and clinical determinants in MDS patients," Blood Cancer Discovery, vol. 2, no. 3, pp. 2643-3230, 2021.

[21] M. Jiang, L. Cheng, F. Qin, L. Du and M. Zhang, "White blood cells classification with deep convolutional neural networks," International Journal of Pattern Recognition and Artificial Intelligence, vol. 32, no. 9, pp. 1857006, 2018.

[22] K. K. Tabe, Y. Ai, T. Ali, I. Takehara and H. Fukuda, "A novel automated image analysis system using deep convolutional neural networks can assist to differentiate MDS and AA," Scientific Reports, vol. 9, pp. 16-22, 2019.

[23] D. Ryu, J. Kim, D. Lim, H. S. Min, I. Y. You et al., "Label-free bone marrow white blood cell classification using refractive index tomograms and deep learning," BME Frontiers, vol. 2021, no. 5, pp. 1-9, 2021. 
[24] Y. Y. Wu, T. C. Huang, R. H. Ye, W. H. Fang, S. W. Lai et al., "A hematologist-level deep learning algorithm (BMSNet) for assessing the morphologies of single nuclear balls in bone marrow smears: Algorithm development," JMIR Medical Informatics, vol. 8, no. 4, pp. e15963, 2020.

[25] D. Kumar, N. Jain, A. Khurana, S. Mittal, S. Satapathy et al., "Automatic detection of white blood cancer from bone marrow microscopic images using convolutional neural networks," IEEE Access, vol. 8, pp. 142521142531, 2020. 